\title{
Vortex simulations on a 3-sphere
}

\author{
O. M. Dix and R. J. Zieve $\odot$ \\ Physics Department, University of California at Davis, Davis, California 95616, USA
}

(Received 25 June 2019; published 24 December 2019)

\begin{abstract}
We study vortex motion using a Hopf flow on a 3-sphere, in place of the standard 3-torus defined by periodic boundary conditions. Although both of these underlying manifolds look locally like $\mathbb{R}^{3}$, the vortex behavior differs significantly. This raises the question of how well calculations on the 3-torus correspond to behavior in $\mathbb{R}^{3}$, for this problem or others. We find that, on both the 3-sphere and 3-torus, vortex tangles appear, with their line density related to the strength of the driving velocity field. However, tangles on the 3-sphere are highly anisotropic, with vortices tending to align along the flow direction. Standard power-law dependences change accordingly from their values in more isotropic tangles. The line length density $\langle L\rangle$ is proportional to $v_{n s}^{1.28}$, where $v_{n s}$ is the drive velocity, and the reconnection rate depends roughly on $\langle L\rangle^{2}$. These compare to $v_{n s}^{2}$ and $\langle L\rangle^{2.5}$ for the more isotropic tangles on the 3-torus. In addition, in simulations using the local induction approximation rather than the full Biot-Savart law, the tangle collapses so that all vortices are nearly aligned with a single flow line. By contrast, vortices on the 3-torus become perpendicular to the driving velocity.
\end{abstract}

DOI: 10.1103/PhysRevResearch.1.033201

\section{INTRODUCTION}

The notoriously intractable equations governing fluid flow generate a great deal of numerical work [1-4]. While many calculations deal directly with the velocity field, others focus on the vorticity [4]. The latter is natural for a variety of flows with intense vorticity, including airplane trailing vortices, severe weather events such as hurricanes and tornadoes, and the molecular clouds that lead to star formation. Vortex methods track the positions of vortex filaments over time, by calculating the velocity field from the vortex locations and then using it to update the vortices. Such calculations are particularly well suited to superfluid helium, where the restriction of vorticity to angstrom-scale cores makes the "slender filament" approximation quite accurate, and a great deal of work has been devoted to understanding such simulations of superfluid vortices $[5,6]$.

Efforts to identify general principles necessarily focus on idealized cases, such as homogeneous turbulence. Since homogeneity is destroyed near a boundary, computations can take two approaches. One is to use a large, finite volume, but to evaluate properties only in a smaller region far from the boundaries. The other is to run calculations in a space without boundaries. The latter has several advantages, notably that there is no need to deal with the complex behavior at surfaces, and that the entire computational power can be devoted to the region of interest. The standard choice is periodic boundary conditions. Topologically, these three-dimensional

Published by the American Physical Society under the terms of the Creative Commons Attribution 4.0 International license. Further distribution of this work must maintain attribution to the author(s) and the published article's title, journal citation, and DOI. simulations run on the three-dimensional torus $T^{3}$ rather than in infinite $\mathbb{R}^{3}$.

The choice of periodic boundary conditions can affect the calculation results. An example encountered in vortex filament simulations is the tendency of vortices to fall into an "open-orbit" state, where they align parallel to each other and perpendicular to the driving velocity field $[7,8]$. In this configuration they interact only trivially with the velocity field, resulting in uniform translation of the entire set of vortices, so the open-orbit state persists indefinitely. Since this state requires the vortices to be closed loops that are perpendicular to the velocity field everywhere, it corresponds to a set of infinite straight vortex lines in $\mathbb{R}^{3}$. Vortex rings in $\mathbb{R}^{3}$, unlike those in $T^{3}$, cannot exhibit such behavior. The particular case of the open-orbit state can be prevented by including nonlocal interaction terms between vortices in addition to the Arms-Hama local term [9], but it nevertheless demonstrates the possibility of topological artifacts. This raises the question of how else the topology may affect simulation results.

Here we present vortex filament simulations in a different three-dimensional space, namely a 3 -sphere $S^{3}$, the surface of a four-dimensional ball. We find several unusual behaviors, including a tendency for vortices to align with the velocity flow instead of perpendicular to it. A particularly interesting result is the unusually high degree of directionality in homogeneous vortex tangles, which provides a testing ground for predictions about anisotropic turbulence.

\section{COMPUTATIONAL CONSIDERATIONS}

As is standard in vortex filament simulations $[5,10]$, we consider a vortex line $\mathbf{s}(\xi, t)$, where $\xi$ is arc length and $t$ is time. The vortex moves according to

$$
\dot{\mathbf{s}}=\dot{\mathbf{s}}_{0}+\alpha \mathbf{s}^{\prime} \times\left(\mathbf{v}_{n s}-\dot{\mathbf{s}}_{0}\right) .
$$


Here $\mathbf{s}^{\prime}=d \mathbf{s} / d \xi$ is the unit tangent to the vortex, $\alpha$ is a coefficient of mutual friction between the normal and superfluid components, and $\mathbf{v}_{n s}$ is the relative velocity of these two components. We take $\alpha=0.1$, corresponding to a temperature of about $1.6 \mathrm{~K}$ in superfluid ${ }^{4} \mathrm{He}$. The complete version of Eq. (1) includes an additional mutual friction term $-\alpha^{\prime} \mathbf{s}^{\prime} \times\left(\mathbf{s}^{\prime} \times\left(\mathbf{v}_{n s}-\dot{\mathbf{s}}_{0}\right)\right)$, but as is often done in helium vortex simulations we set the coefficient $\alpha^{\prime}=0$ and neglect this contribution. Finally, $\dot{\mathbf{s}}_{0}$ is the local superfluid velocity, given by the Biot-Savart law. In $\mathbb{R}^{3}$ this is

$$
\dot{\mathbf{s}}_{0}=\frac{\kappa}{4 \pi} \int_{\mathscr{L}} \frac{\left(\mathbf{s}_{1}-\mathbf{s}\right) \times d \mathbf{s}_{1}}{\left|\mathbf{s}_{1}-\mathbf{s}\right|^{3}},
$$

where $\kappa$ is the circulation quantum and the integral is over all the vortex lines. We will discuss below the modifications needed to this integral for use in $S^{3}$. Our choice of friction parameters and our use of a constant applied normal fluid flow match various vortex filament calculations on $T^{3}$ exactly $[7,11,12]$ or nearly $[9,13]$, so we can compare our results with these simulations. The corresponding physical situation is somewhere between $\mathrm{T} 1$ and $\mathrm{T} 2$ counterflow turbulence. T1 involves laminar normal flow but with a nonconstant profile due to the channel walls, while T2 has turbulent normal flow with the average normal velocity independent of position in the channel [14-16].

For numerical evaluation, the vortex is approximated by a sequence of points with straight line segments connecting neighboring points. In evaluating the velocity at $\mathbf{s}$, the two vortex segments adjacent to $\mathbf{s}$ must be removed from the integral. They are replaced by a term proportional to the local curvature of the vortex line, which is scaled to agree with the analytically calculated velocity for circular vortex rings. The expression for local superfluid velocity becomes

$$
\dot{\mathbf{s}}_{0}=\beta \mathbf{s}^{\prime} \times \mathbf{s}^{\prime \prime}+\frac{\kappa}{4 \pi} \int_{\mathscr{L}}^{\prime} \frac{\left(\mathbf{s}_{1}-\mathbf{s}\right) \times d \mathbf{s}_{1}}{\left|\mathbf{s}_{1}-\mathbf{s}\right|^{3}},
$$

where $\mathbf{s}^{\prime \prime}=d^{2} \mathbf{s} / d \xi^{2}$ is the curvature vector and the primed integral denotes the exclusion of the adjacent segments. The prefactor $\beta$ is set to

$$
\beta=\frac{\kappa}{4 \pi} \ln \left(\frac{2 \sqrt{\ell_{+} \ell_{-}}}{e^{1 / 4} a_{0}}\right),
$$

which yields the correct behavior for circular vortex loops in $\mathbb{R}^{3}$ with uniform point spacing. $a_{0}$ is the core radius of a vortex filament, and $\ell_{ \pm}$are the distances between the point where the velocity is being calculated and its neighbors on each side. Our calculations use values appropriate to superfluid ${ }^{4} \mathrm{He}, \kappa=$ $9.969 \times 10^{-4} \mathrm{~cm}^{2} / \mathrm{s}$ and $a_{0}=1.3 \times 10^{-8} \mathrm{~cm}$.

We use a 3 -sphere embedded in $\mathbb{R}^{4}$, consisting of points $(x, y, z, w)$ such that $x^{2}+y^{2}+z^{2}+w^{2}=R^{2}$. (Mathematicians often use $S^{3}$ for the sphere of unit radius, but we will use this notation more generally for our sphere of radius $R=$ $0.005 \mathrm{~cm}$.) Four-dimensional Cartesian coordinates help with much of the necessary calculation. For the driving velocity, we use a Hopf vector field, given in $\mathbb{R}^{4}$ as $\mathbf{v}=\frac{v}{R}(-y, x,-w, z)$. This Hopf velocity field possesses several useful properties. It is tangent to $S^{3}$, so it drives vortex motion entirely within the manifold. It also has uniform magnitude $v$ and zero divergence. However, it is not irrotational; in fact its curl in
$S^{3}$ is $\frac{2}{R} \mathbf{v}$, parallel to the Hopf field itself [17]. We assign the driving Hopf velocity field to the normal fluid, which unlike the superfluid is not required to be irrotational. Nonetheless, as we shall see, the nonzero curl has consequences for the vortex behavior. With the applied superfluid velocity set to zero, we have $\mathbf{v}_{n s}=\mathbf{v}$.

Several adjustments are needed when restricting to $S^{3}$. To begin with, distances such as $\left|\mathbf{s}_{1}-\mathbf{s}\right|$ should be calculated along the geodesic through the two points, rather than along the shorter chord that connects the points in $\mathbb{R}^{4}$. Next, vectors defined at a point on $S^{3}$ lie in the tangent space of $S^{3}$ at that point. Vector operations such as dot products or cross products can be performed within a tangent space. However, in our calculations relevant vectors are often defined at two different points of $S^{3}$, where the tangent spaces themselves differ. Viewed as a vector within $\mathbb{R}^{4}$, one vector may not even be in the tangent space where the other vector is defined. For vectors in different tangent spaces, we parallel transport one vector along a geodesic to the location of the other vector, before carrying out any further vector operations [18]. Once we are working with two vectors in the same tangent space, the dot product takes exactly its value in $\mathbb{R}^{4}$. For cross-products in the tangent space at $\mathbf{q}$, we use the determinant of a $4 \times 4$ matrix:

$$
\operatorname{cross}\left(\mathbf{v}_{1}, \mathbf{v}_{2}, \mathbf{q}\right) \cdot \mathbf{a}=\frac{1}{R}\left|\begin{array}{llll}
v_{1 x} & v_{1 y} & v_{1 z} & v_{1 w} \\
v_{2 x} & v_{2 y} & v_{2 z} & v_{2 w} \\
q_{x} & q_{y} & q_{z} & q_{w} \\
a_{x} & a_{y} & a_{z} & a_{w}
\end{array}\right|
$$

Here $\mathbf{a}$ is an arbitrary vector in $\mathbb{R}^{4}$; taking it to be a unit vector selects the component of the cross-product in that direction. Given three vectors $\mathbf{v}_{1}, \mathbf{v}_{2}$, and $\mathbf{q} / R$, the cross-product is a vector orthogonal to all three and with magnitude defined by the volume of the parallelepiped spanned by the three vectors. Since $\mathbf{q}$ is the normal vector to the 3 -sphere, the cross-product must lie in the tangent space at $\mathbf{q}$.

Some of the vectors involved in the calculations, particularly the curvature vectors of vortex lines as calculated in $\mathbb{R}^{4}$, may have components along the 3 -sphere radius vector. Such radial components give no contribution to cross-products, but they could affect dot products. We explicitly remove any radial component of a vector before proceeding with further operations. In the case of curvature vectors, this has the effect of removing the intrinsic curvature of the 3 -sphere from our calculations.

Another issue is that numerical evaluation of Eq. (1) yields a velocity vector in a tangent space to $S^{3}$, not in $S^{3}$ itself. Using such velocity vectors to update the locations of vortex core would result in new locations outside the 3 -sphere. Projecting these points directly back to the 3 -sphere would slightly decrease the distance traveled during the time step. Instead, we move the vortex points by the desired distance but along the geodesic defined by the projection of the velocity onto $S^{3}$.

The Biot-Savart law must also be treated differently on $S^{3}$ from on $T^{3}$. The 3-torus has no curvature, so within regions very small compared to the 3-torus diameters, the Biot-Savart expression of $\mathbb{R}^{3}$ can be used. As the distance between the vortex segment and the test point grows, additional contributions enter corresponding to paths that loop around the torus, as shown in Fig. 1 for $T^{2}$. The shortest path between any part 


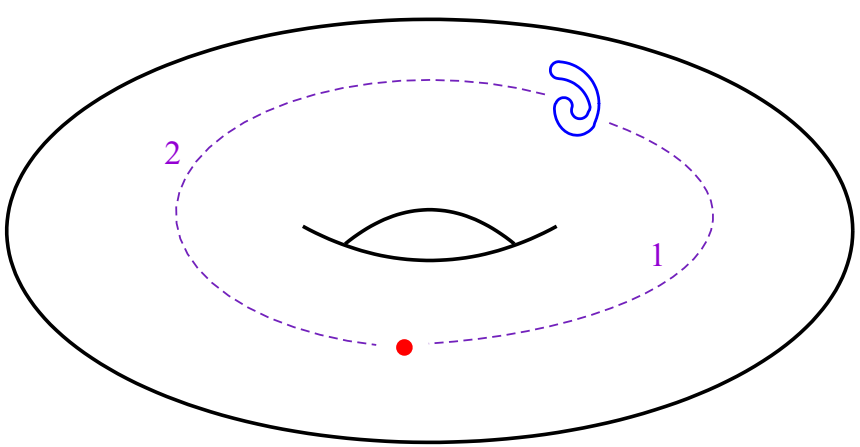

FIG. 1. Two possible paths from a vortex loop (blue) to the same point (red) on a torus.

of the vortex and the point indicated lies near the dotted line 1; in the analogous three-dimensional situation, this would give certain Biot-Savart contributions to the velocity field at the test point. On the other hand, the dotted line 2 corresponds to paths not much longer but in an entirely different direction, which would give very different contributions. Other paths, wrapping around the torus one or more times, are also possible. The multiple contributions have sometimes been accounted for by representing the 3 -torus as a periodic cube tiling $\mathbb{R}^{3}$, and adding contributions from the original vortex segment and also from its images, out to some distance beyond which further contributions are deemed negligible [13].

On $S^{3}$ the standard Cartesian Biot-Savart expression is again merely an approximate solution, both because alternate paths may traverse the 3 -sphere in different directions and because the curvature of the 3-sphere makes the resemblance to $\mathbb{R}^{3}$ only local. Some of our computations use the standard Euclidean Biot-Savart law within a limited region of $S^{3}$. To update the position of a point $P$ along the vortex core, we need to calculate the fluid velocity at that point. We use parallel transport to map all vectors needed for the calculation into the tangent space at $P$. We then carry out the calculation within that tangent space. We calculate contributions only from vortex segments sufficiently close to $P$. In Sec. III we refer to this method as an "approximate Biot-Savart calculation."

Most of our calculations use a different approach. Unlike on $T^{3}$, there exists an exact form for the Biot-Savart law on $S^{3}[19,20]$, which allows a more complete calculation of the velocity field produced by vortex segments. We generally use the full 3-sphere Biot-Savart law. We retain contributions to velocity either from vortices on the entire 3-sphere or from those in a limited region surrounding the evaluation point. Whether we use the Cartesian Biot-Savart law or the exact expression on $S^{3}$, we project the calculated velocity vector onto a geodesic and move $P$ an appropriate distance along that geodesic.

We follow the presentation by DeTurck and Gluck of the Biot-Savart law on the 3-sphere [19]. The contribution to the velocity field at a point $\mathbf{q}$ from a vortex line $U$ on $S^{3}$ is given by

$$
d \dot{\mathbf{s}}_{0}=\int_{U} \nabla_{\mathbf{q}} \phi(\mathbf{u}, \mathbf{q}) \times P_{\mathbf{q u}} d \mathbf{u} .
$$

Here $P_{\mathbf{q u}}$ is the parallel transport operation described above, which takes a vector from the tangent space at $\mathbf{u}$ to the tangent

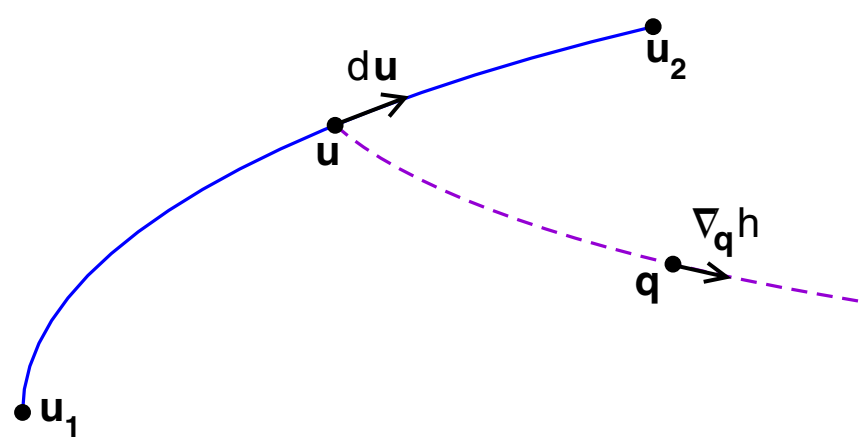

FIG. 2. Integration along a geodesic (solid curve) to evaluate Biot-Savart contribution at point $\mathbf{q}$. The dashed curve is the geodesic between $\mathbf{q}$ and a point $\mathbf{u}$ on the integration path.

space at q. The function $\phi$ depends only on the distance $h=$ $|\mathbf{q}-\mathbf{u}|$ along the geodesic connecting its two arguments and has the form

$$
\phi(h)=\frac{\kappa}{4 \pi^{2} R^{2}}\left(\pi-\frac{h}{R}\right) \csc \frac{h}{R} .
$$

The familiar Biot-Savart law in $\mathbb{R}^{3}$ would instead use $\phi(h)=$ $\kappa / 4 \pi h$, with $h$ the distance along the straight-line path from $\mathbf{q}$ to $\mathbf{u}$.

For the Biot-Savart calculation we consider the vortex core as a series of points connected by straight segments, i.e., geodesics. We evaluate the integral of Eq. (2) exactly along each of these finite-length geodesic segments, as shown in Fig. 2. In the integrand, $\nabla \phi$ is calculated in the tangent space at q. Since $\phi$ depends only on the separation between the points $\mathbf{u}$ and $\mathbf{q}$, the gradient must be in the direction where this separation changes the fastest, which is the direction of the geodesic containing the two points. We use

$$
\nabla_{\mathbf{q}} \phi=\frac{\partial \phi}{\partial h} \nabla_{\mathbf{q}} h
$$

and observe that $\nabla_{\mathbf{q}} h$ has magnitude 1 and is directed away from $\mathbf{u}$ along the geodesic connecting $\mathbf{u}$ and $\mathbf{q}$.

Next consider the effect of parallel transport along this geodesic. The geodesic lies in a plane of $\mathbb{R}^{4}$, defined by the tangent to the geodesic and the normal to the 3 -sphere at any point on the geodesic, such as $\mathbf{u}$. Parallel transport of any vector rotates the vector within this plane, without affecting the components orthogonal to the plane. This has two implications for the calculation at hand. First, no parallel transport calculation is necessary for the integral of Eq. (2). Only the components of $P_{\mathbf{q u}} d \mathbf{u}$ within the tangent space but perpendicular to the geodesic contribute to the crossproduct in the integrand, and these are exactly the components unchanged by parallel transport. Second, we can use u to construct a unit vector along the geodesic. The plane of a geodesic contains the normal vectors to $S^{3}$ at every point on that geodesic, so $\mathbf{u}$ can be decomposed into one component along $\mathbf{q}$ and another tangent to the geodesic at $\mathbf{q}$. Its projection into the tangent space at $\mathbf{q}$ is this latter component. Since the angle between $\mathbf{u}$ and $\mathbf{q}$ is $h / R$, the two components have magnitude $R \cos \frac{h}{R}$ and $R \sin \frac{h}{R}$. Rescaling, the projection of $-\frac{1}{R \sin (h / R)} \mathbf{u}$ into the tangent space at $\mathbf{q}$ is a unit vector along the geodesic, directed away from u. For Eq. (2) we can use 
$-\frac{1}{R \sin (h / R)} \mathbf{u}$ rather than $\nabla_{\mathbf{q}} h$; although these vectors are not identical, the component of the former perpendicular to the tangent space does not contribute to the cross-product. Using this fact and explicitly calculating $\partial \phi / \partial h$, Eq. (2) becomes

$$
d \dot{\mathbf{s}}_{0}=\frac{\kappa}{4 \pi^{2} R^{3}} \int_{\mathbf{u}_{1}}^{\mathbf{u}_{2}} d \mathbf{u} \times \mathbf{u} \frac{\sin \frac{h}{R}+\left(\pi-\frac{h}{R}\right) \cos \frac{h}{R}}{\sin ^{3} \frac{h}{R}} .
$$

Up to this point the discussion does not depend on the shape of the integration path from $\mathbf{u}_{1}$ to $\mathbf{u}_{2}$. We now take that path to be a geodesic. This is distinct from the geodesic of the previous paragraph, which goes from the test point to the integration path. Next we set coordinates for evaluating the integral. The geodesic integration path defines a plane in $\mathbb{R}^{4}$, which we take as the $x y$ plane. We take the projections of $\mathbf{q}$ within and perpendicular to the $x y$ plane to lie along the positive $x$ and $z$ axes, respectively. Thus $\mathbf{u}=R(\cos \theta, \sin \theta, 0,0)$, with $\theta_{1}$ and $\theta_{2}$ denoting the integration limits, and $\mathbf{q}=R(\cos \psi, 0, \sin \psi, 0)$, where $0 \leqslant \psi \leqslant$ $\pi / 2$. Hence $d \mathbf{u}=R(-\sin \theta, \cos \theta, 0,0) d \theta$ and the integral becomes

$$
d \dot{\mathbf{s}}_{0}=-\frac{\kappa}{4 \pi^{2}} \hat{\mathbf{w}} \int_{\theta_{1}}^{\theta_{2}} d \theta \sin \psi \frac{\sin \frac{h}{R}+\left(\pi-\frac{h}{R}\right) \cos \frac{h}{R}}{\sin ^{3} \frac{h}{R}},
$$

where $h$ depends on $\theta$ and $\hat{\mathbf{w}}$ is a unit vector in the positive $w$ direction. We can also adjust the integral so that $\sin \theta \geqslant 0$, as follows. If the original choice of coordinates gives $\sin \theta \leqslant 0$ along the entire geodesic, then rotation by $\pi$ in the $y w$ plane switches the geodesic segment to positive $\theta$ without altering q. If $\sin \theta$ changes sign along the geodesic, then the curve can be divided into two segments and the calculation carried out separately for the two. The geodesic segments we integrate along connect consecutive points on a vortex core and are always too short for $\sin \theta$ to change sign more than once.

The variables $\theta, \psi$, and $h$ are related through

$$
\cos \frac{h}{R}=\frac{\mathbf{u} \cdot \mathbf{q}}{R^{2}}=\cos \theta \cos \psi .
$$

Using $-\frac{1}{R} \sin \frac{h}{R} d h=-\sin \theta \cos \psi d \theta$ and assuming $\sin \theta \geqslant$ 0 , we have

$$
d \dot{\mathbf{s}}_{0}=-\frac{\kappa \sin \psi}{4 \pi^{2} R} \hat{\mathbf{w}} \int_{h_{1}}^{h_{2}} d h \frac{\sin \frac{h}{R}+\left(\pi-\frac{h}{R}\right) \cos \frac{h}{R}}{\sin ^{2} \frac{h}{R} \sqrt{\cos ^{2} \psi-\cos ^{2} \frac{h}{R}}} .
$$

An additional minus sign would appear if $\sin \theta \cos \psi<0$, but our choice of coordinates ensures that this is not the case. Integrating gives

$$
\begin{aligned}
d \dot{\mathbf{s}}_{0}= & \hat{\mathbf{w}} \frac{\kappa \sin \psi}{4 \pi^{2}\left(\cos ^{2} \psi-1\right)}\left(-\arcsin \left(\frac{\cos \frac{h}{R}}{\cos \psi}\right)\right. \\
& \left.+\frac{\left(\pi-\frac{h}{R}\right) \sqrt{\cos ^{2} \psi-\cos ^{2} \frac{h}{R}}}{\sin \frac{h}{R}}\right)\left.\right|_{h_{1}} ^{h_{2}},
\end{aligned}
$$

where our coordinates again eliminate any ambiguity in the sign of the first term. In the coordinates used above for the geodesic segment, the contribution is always in the $\mathbf{w}$ direction. We then rotate it back into the original coordinate frame in which the vortices are defined, repeat for each geodesic (a)

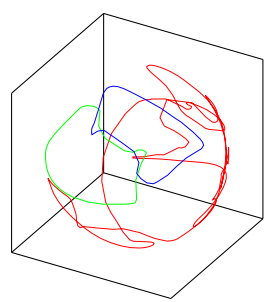

(c)

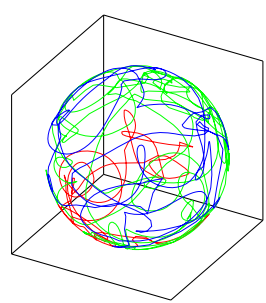

(e)

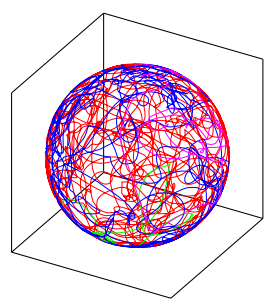

(b)

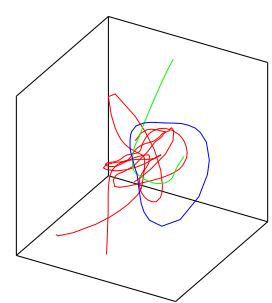

(d)

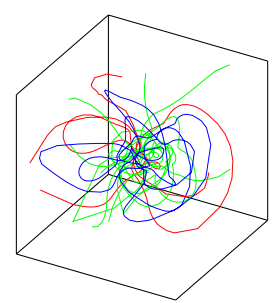

(f)

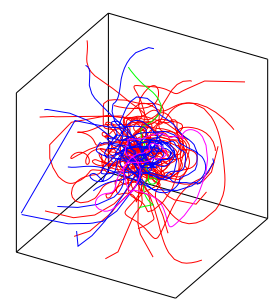

FIG. 3. Hopf (left) and stereographic (right) projections of steady-state vortex tangles for $v_{n s}=1,2,4 \mathrm{~cm} / \mathrm{s}$ on 3 -sphere of radius $R=0.005 \mathrm{~cm}$.

segment along the vortices, and add the results to obtain the Biot-Savart integral along the entire set of vortices.

In addition to the differential equation (1), a separate treatment is needed when two vortex segments approach each other closely. Direct numerical simulations [21,22] confirm that in most such cases the vortices form cusps that draw ever closer to each other. A standard feature of vortex filament calculations is to perform reconnections [5,12,13,23], swapping how the heads and tails of the two segments connect. Reconnection is also observed experimentally in classical fluids [24,25] and in superfluid helium [26]. We carry out reconnections once vortices approach to within a cutoff separation. The cutoff is usually a fraction of the local radius of curvature, but to prevent nearly straight vortices from reconnecting at large distances we also impose an absolute maximum on the cutoff separation. We then set up the reconnection itself to conserve vortex line length.

\section{PROPERTIES OF STABLE TANGLES}

Calculations using these equations successfully produce vortex tangles, as shown in Fig. 3. All our simulations use $R=0.005 \mathrm{~cm}$. To display the vortex configuration we use two types of projection, Hopf and stereographic. These are described in more detail in the Appendix, as well as in topology textbooks [27]. The right column of Fig. 3 uses stereographic projection, which maps $S^{3}$ onto flat three-dimensional space $\mathbb{R}^{3}$. One point of $S^{3}$ goes to infinity and its antipode maps to the origin. A sphere within $S^{3}$, centered at either of these 


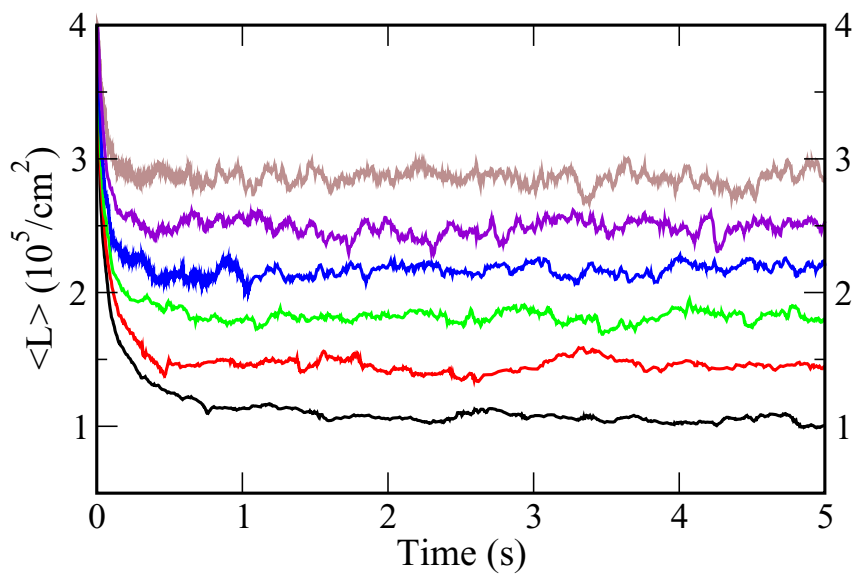

FIG. 4. Line length density as a function of time, for (bottom to top) $v_{n s}=1,1.2,1.4,1.6 .1 .8,2 \mathrm{~cm} / \mathrm{s}$. The starting point in each case is the same highly distorted vortex loop.

points, maps to a sphere in $\mathbb{R}^{3}$ centered at the origin. Hence the half of $S^{3}$ closest to the point sent to the origin maps to a ball centered at the origin. The other half of $S^{3}$ maps to the remainder of $\mathbb{R}^{3}$ outside of the ball. Some portions of the vortex lines in Fig. 3 lie outside the displayed region. The second type of visualization, shown in the left column of Fig. 3, is the Hopf projection, which maps $S^{3}$ onto $S^{2}$, the two-dimensional surface of a ball in $\mathbb{R}^{3}$. Each Hopf fiber maps to a single point of $S^{2}$. This mapping is particularly informative when vortices align closely with the velocity field, since after projection such vortices appear as points or very small loops.

As seen previously in many calculations using periodic boundary conditions [7-9,11,13,23], the vortex tangle eventually reaches a steady state where various statistical properties remain constant over time. Figure 4 shows the time development of the average vortex line density, for several different driving velocities. The flat portion of each curve corresponds to the steady-state situation. As the driving velocity $v_{n s}$ increases, the line density increases monotonically and the time to reach the steady-state density decreases.

We find that the statistical behavior of tangles is independent of the initial conditions for the calculations, as shown in Fig. 5 for initial setups that differ by more than a factor of five in vortex line length. Each of the initial setups is a single highly distorted vortex. They are generated by starting with a single circular vortex in the $x y$ plane with a sinusoidal distortion in the perpendicular directions. We run the code to generate a tangle, although not necessarily under the same conditions as in Fig. 5. We then select a single vortex line from the tangle, which becomes the starting point of Fig. 5. The motivation is to remove any symmetry from the initial configuration.

For further verification that our new code with the exact Biot-Savart formula for $S^{3}$ functions properly, we compare calculations with the more familiar, approximate Biot-Savart law. As noted earlier, this approximation should be very good when the distances involved are much shorter than the 3sphere radius. Simulations using the exact or approximate formulas indeed agree well for dynamics of simple vortex

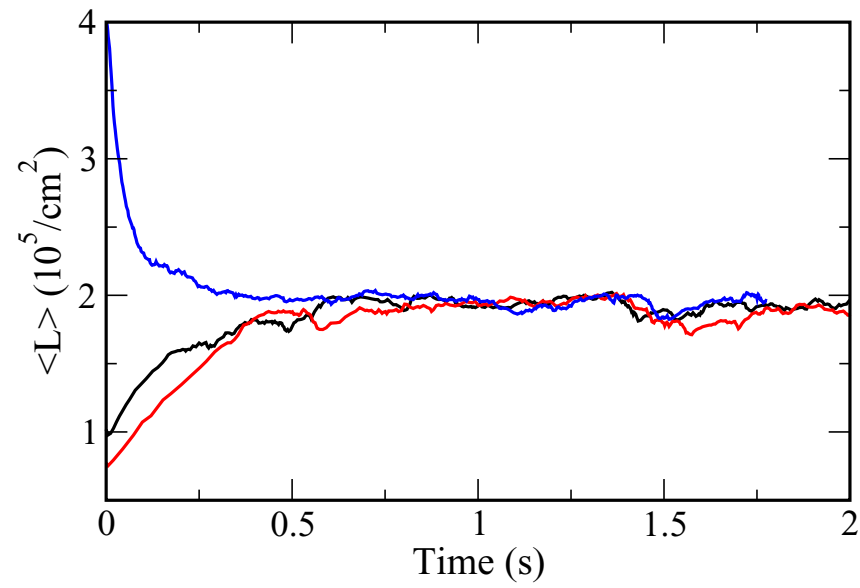

FIG. 5. Line length density $\langle L\rangle$ as a function of time, for $v_{n s}=$ $1.4 \mathrm{~cm} / \mathrm{s}$ and nonlocal interactions calculated within a hemisphere, for three different initial configurations. Each initial configuration consists of a single distorted vortex loop, albeit with very different total length. The two calculations with initially increasing $\langle L\rangle$ use the exact Biot-Savart law, while the third uses the approximate BiotSavart formula.

configurations, such as a single circular vortex ring. Their results for line length density and tangle anisotropy also agree, not only for very short-distance calculations of vortex interactions, but even when applied on as much as a full hemisphere. Thus Fig. 5 includes two curves using the exact Biot-Savart law and a third using the approximate version, with the line length density equilibrating at the same level for all three. In each case vortex interactions out to a hemisphere were included. We do not attempt to use the approximate Biot-Savart law at larger distances.

Despite the rapid achievement of a steady-state line density in Fig. 4, the tangles, particularly for low $v_{n s}$, are not entirely homogeneous. Figure 6 shows the line density in four of the sixteen orthants of $S^{3}$. Although the line density is very similar in certain pairs of orthants, in others it differs significantly. We number the orthants by assigning one bit to each of the four Cartesian coordinates of the space $\mathbb{R}^{4}$ that contains the 3 -sphere. A negative coordinate corresponds to a bit value of zero, and a positive or zero coordinate to a value of one. We place the $x$ bit in the rightmost position, then the $y$ bit, then $z$, and finally the $w$ bit in the leftmost position. Thus orthant 1 has $x \geqslant 0, y<0, z<0$, and $w<0$, while orthant 13 has $x \geqslant$ $0, y<0, z \geqslant 0$, and $w \geqslant 0$. As Fig. 7 shows, the line densities in certain pairs of orthants continue to diverge for several seconds, even though the long-time average line density is the same in both orthants. The issue is not a slow approach to the steady-state line density, but rather slow communication between different regions of $S^{3}$ even in the steady state.

The top frame of Fig. 8 shows the line length density for all sixteen orthants, averaged over the same four seconds of steady-state tangle shown in Fig. 6. The orthants separate into four groups by line density. Orthants $0,5,10$, and 15 have by far the largest line density. The next group is orthants $1,7,8$, and 14 , then orthants $2,4,11$, and 13 with nearly identical line length densities, and finally orthants $3,6,9$, and 12 . These groupings track the velocity field. For example, 


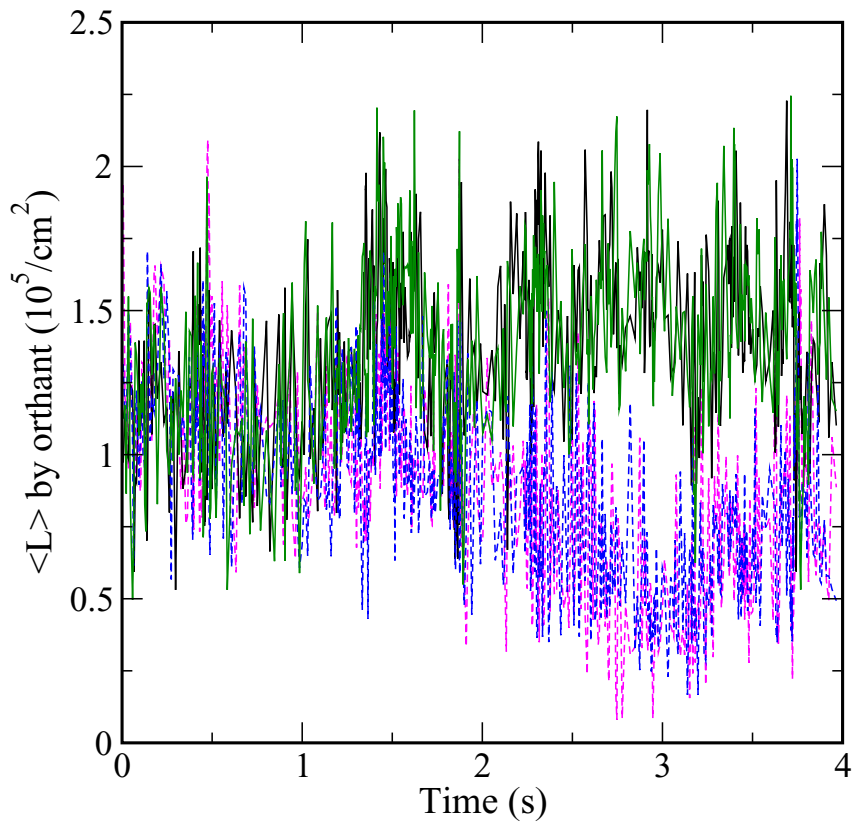

FIG. 6. Line length density as a function of time for $v_{n s}=$ $1 \mathrm{~cm} / \mathrm{s}$, for orthants 9 (magenta, dashed), 10 (black, solid), 12 (blue, dashed), and 15 (green, solid). The two solid curves track each other closely, as do the two dashed curves, showing rapid homogenization in the corresponding orthants.

consider the point $\left(-\frac{1}{2},-\frac{1}{2},-\frac{1}{2},-\frac{1}{2}\right)$, which lies exactly in the center of orthant 0 . The flow line through this point also passes through quadrants 5,10 , and 15 ; in fact, it reaches the points $\left(\frac{1}{2},-\frac{1}{2}, \frac{1}{2},-\frac{1}{2}\right),\left(\frac{1}{2}, \frac{1}{2}, \frac{1}{2}, \frac{1}{2}\right)$, and $\left(-\frac{1}{2}, \frac{1}{2},-\frac{1}{2}, \frac{1}{2}\right)$ at the centers of these orthants. Likewise the centers of orthants

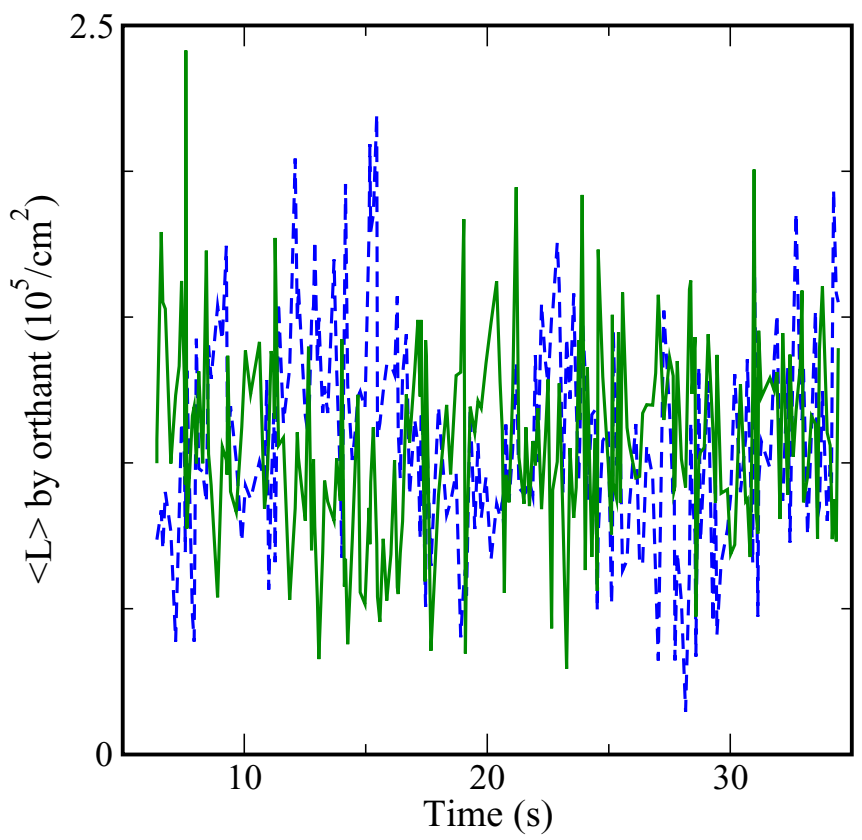

FIG. 7. Continuation of line length density from Fig. 6, for orthants 12 (blue, dashed) and 15 (green, solid). Over sufficiently long times the average density is the same in each orthant. For clarity the sampling rate here is much lower than in Fig. 6.

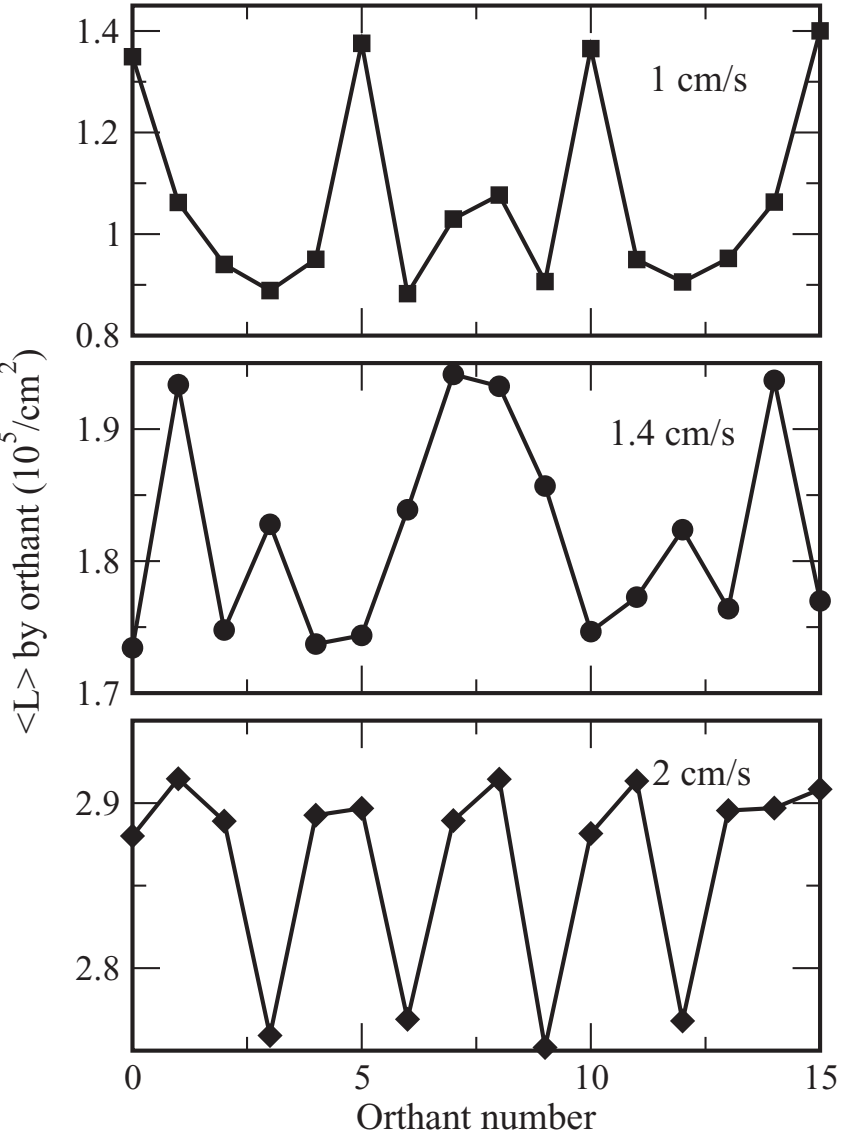

FIG. 8. Line length density for the sixteen orthants of $S^{3}$, for steady-state tangles with $v_{n s}=1 \mathrm{~cm} / \mathrm{s}$ (top), $v_{n s}=1.4 \mathrm{~cm} / \mathrm{s}$ (center), and $v_{n s}=2 \mathrm{~cm} / \mathrm{s}$ (bottom). In each case the average is over a 4-s interval.

$1,7,8$, and 14 lie on a common flow line, and similarly for the remaining two sets of orthants. A generic flow line actually passes through eight orthants, two of the listed sets of four. The portion of the flow line within an orthant is always identical for the four orthants in a set. In particular, flow lines which pass through a large portion of one orthant do the same for the other orthants in the same group, alternating with briefer sections in the orthants of a second group. Hence it makes sense to think of the velocity field as roughly conveying any vortex tangle from one orthant into the other orthants grouped with it. The implication for homogeneity is that when vortices are swept directly from one orthant to another through the influence of the applied velocity field, the line length in those orthants quickly equilibrates. The process is much slower for orthants in different groups, which do not benefit from this convective mechanism. The resulting lack of global homogeneity for tangles on $S^{3}$ differs from the situation on $T^{3}$, where vortex tangles remain homogeneous. As we will see shortly, the reason may be the different anisotropy of tangles in the two spaces.

The same presentation for higher driving velocities appears in the bottom two frames of Fig. 8. The same groupings of orthants appear, since that depends solely on the geometry of $S^{3}$. However, at each velocity different sets of orthants have the largest and smallest line densities. As the velocity 


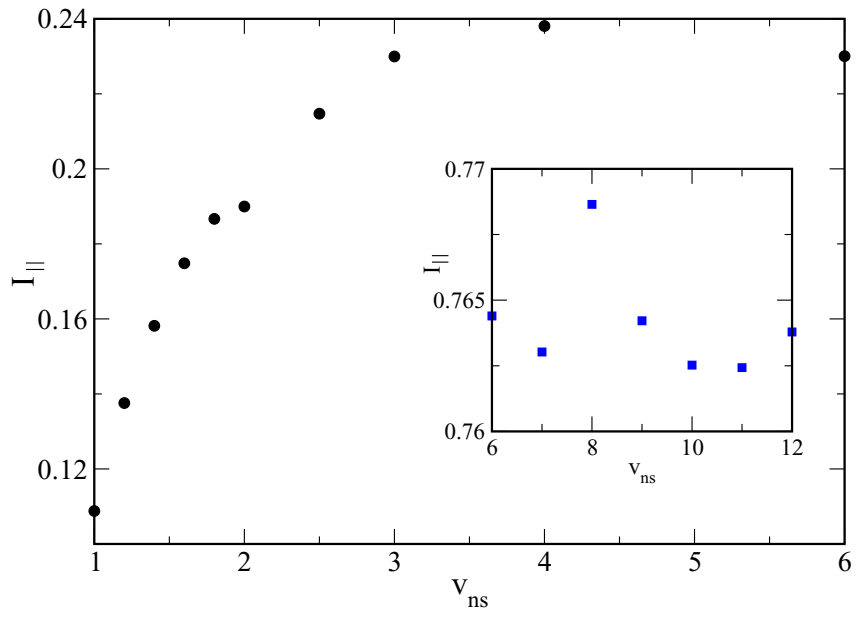

FIG. 9. Anisotropy parameter $I_{\|}$as a function of applied velocity. For an isotropic tangle, $I_{\|}=2 / 3$. Inset: anisotropy parameter from 3torus simulations, using periodic boundary conditions on a cube with side $0.005 \mathrm{~cm}$. The larger 3-torus velocities are roughly equivalent to those used on $S^{3}$. They reflect that a cube of side $0.005 \mathrm{~cm}$ is smaller than a sphere of radius $0.005 \mathrm{~cm}$. In both cases the minimum velocity is the lowest that sustains a vortex tangle.

increases, the tangle becomes more homogeneous, with variations in $\langle L\rangle$ decreasing in both relative and absolute terms. At $v_{n s}=2 \mathrm{~cm} / \mathrm{s}$, three sets of orthants have indistinguishable line densities, with the variations among orthants within a set larger than any differences in line density between sets. The fourth set of orthants has a smaller line density than the other three, but only by about $5 \%$.

A complication in comparing results on $T^{3}$ and $S^{3}$ is how to adjust length scales. For a fixed geometry, a known scaling [5] relates calculations in systems of different sizes. When a torus and sphere have the "same" size is not clear. One option is to require them to have the same volume. Others are to equate the maximum point separation or the average point separation. Since we do not have a solution to this problem, we will not attempt any rescaling between the two shapes. No such difficulty arises in other quantitative comparisons between topologies, such as the exponents in various powerlaw behaviors.

In simulations on $T^{3}$, vortices have a slight tendency to align perpendicular to the applied velocity field. We use the anisotropy parameter [7]

$$
I_{\|}=\frac{1}{V L} \int\left[1-\left(\hat{\mathbf{s}}^{\prime} \cdot \hat{\mathbf{r}}_{\|}\right)^{2}\right] d \xi
$$

to quantify this effect. Here $\hat{\mathbf{r}}_{\|}$is a unit vector parallel to the applied flow, $V L$ is the total length of vortices, and the integral is taken over all vortex lines. If the vortices were entirely aligned with the applied velocity field, then $I_{\|}$would vanish. In the other extreme, with vortices always perpendicular to the applied field, $I_{\|}=1$. In a perfectly isotopic tangle $I_{\|}=\frac{2}{3}$. Our previous calculations on $T^{3}$ give $I_{\|}=0.76$ [11]. Other simulations $[7,9,12,13]$ find similar values for $I_{\|}$ at $\alpha=0.1$, with a decrease towards $2 / 3$ as $\alpha$ decreases. As shown in Fig. 9, the vortex orientation within the 3-sphere tangles is dramatically different. $I_{\|}$lies between 0.1 and 0.25 ,

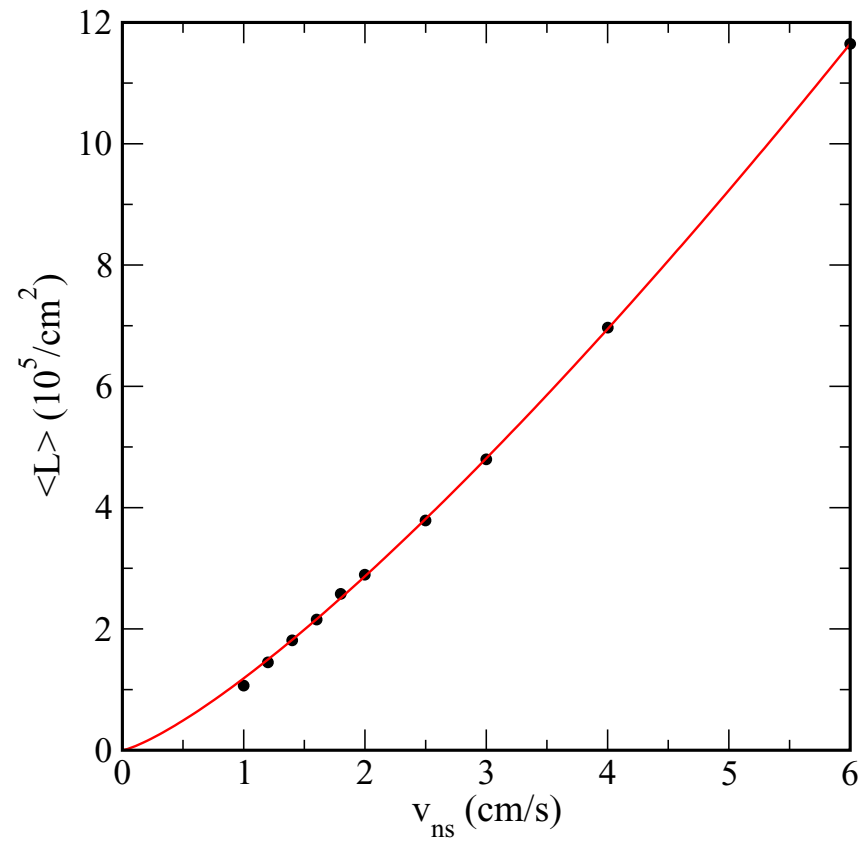

FIG. 10. Line length density as a function of applied velocity. Solid line is a two-parameter fit, yielding $1.19 v_{n s}^{1.28}$, where only velocities $v_{n s} \geqslant 2$ are used for the fit. For comparison, isotropic homogeneous tangles should have an exponent of 2 , close to that observed in 3-torus simulations.

depending on the driving velocity. At sufficiently high velocity the anisotropy levels off at about 0.23 , implying a significant alignment of vortices with the applied velocity.

Alignment of vortices along flow lines limits the effectiveness of convection at homogenizing a tangle. Vortices move roughly parallel to their own direction, and the flow does not force them into interaction with other vortices. By contrast, a vortex locally perpendicular to the velocity field sweeps through a region of space, making vortex crossings more likely. Such a vortex also drags its more distant portions along with it, increasing communication among all parts of the tangle.

The misalignment of the vortices remains large enough to sustain vortex-vortex interactions. Thus polarized turbulence arises naturally on $S^{3}$ with a Hopf driving field. The structure is entirely different from that of counterflow turbulence on $T^{3}$, where the polarization is far less and is directed perpendicular to the flow. Anisotropic turbulence in physical experiments can arise from rotation [28], the geometry of the container boundaries, or entrainment by normal fluid turbulence [29]. Boundaries and a turbulent normal fluid are both computationally expensive, but simulations on $S^{3}$ provide another method of achieving highly polarized turbulence. This may prove especially important in light of evidence that anisotropy persists down to small-scale structures in classical [30-32] and superfluid turbulence [33,34]. If some amount of anisotropy pervades turbulent systems, then understanding how that anisotropy influences the behavior will be necessary.

One illustration of the difference between isotropic and anisotropic turbulence is the dependence of the vortex line density on the applied velocity field. Scaling arguments [7,35] 

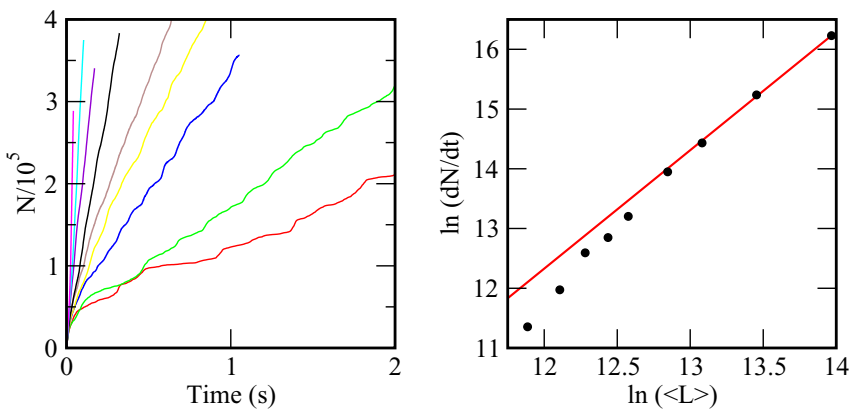

FIG. 11. Left: number of reconnections $N$ as a function of time, for several different velocities. Right: relation between reconnection rate and line length density. Each point corresponds to one of the curves in the left graph, with $d N / d t$ obtained from a linear fit to the long-time part of the $N(t)$ curve. The solid line represents a powerlaw fit, $d N / d t=c\langle L\rangle^{m}$, to the highest four points, selected because the corresponding tangles have similar anisotropy. The resulting exponent is $m=1.98$. By contrast, torus simulations yield a value close to the 2.5 expected from theory.

give a clear prediction for isotropic homogeneous tangles: $\beta\langle L\rangle^{1 / 2}$ should be proportional to $v_{n s}$. This has been observed in experiment [36] and simulations [7,9,13]. The scaling breaks down when rotation introduces anisotropy [28,37]. In these studies the anisotropy parameter changes with $v_{n s}$, obscuring the dependence of $\langle L\rangle$ on $v_{n s}$. For our system, Fig. 10 shows the line length after equilibration at each velocity, along with a power-law fit. Rather than finding that $\langle L\rangle$ goes roughly as $v_{n s}^{2}$, we find an exponent of only 1.28 . The reduced exponent is not caused by the lack of global homogeneity. For the fit shown in Fig. 10 we use only those trials with velocity at least $2 \mathrm{~cm} / \mathrm{s}$, which are nearly homogeneous. Extrapolating the fit curve to lower velocities gives excellent agreement with the line densities found at these low driving velocities, and the fit itself changes little when these points are included. Thus we attribute the change in exponent to the anisotropy of the tangle; certainly anisotropy invalidates the scaling arguments used to derive the quadratic dependence of line density on $v_{n s}$ in isotropic tangles. In fact a reduced exponent makes intuitive sense. Vortices perfectly aligned with the driving velocity cannot gain energy from the applied fields; the $\hat{\mathbf{s}}^{\prime} \times \mathbf{v}_{\mathbf{n}}$ part of the friction term vanishes. Line length increase can only originate from nonaligned vortices, and since these are underrepresented in our tangles, our line length is less than in the isotropic case.

The rate of vortex reconnections can also depend on the structure of the tangle. For isotropic homogeneous turbulence, simple arguments $[38,39]$ show that the reconnection rate is related to the vortex line density through $d N / d t \propto\langle L\rangle^{5 / 2}$, which agrees with 3-torus simulations [12,38]. Polarization should reduce the number of reconnections [40], since aligned vortices encounter each other less often. Simulations show a reduction of about a factor of two in the number of reconnections in polarized turbulence [41]; here the polarization comes from a normal fluid driving velocity taken from a snapshot of classical turbulence. Polarization is also expected to decrease the scaling exponent. In calculations of counterflow turbulence before the vortex line length stabilized [10],

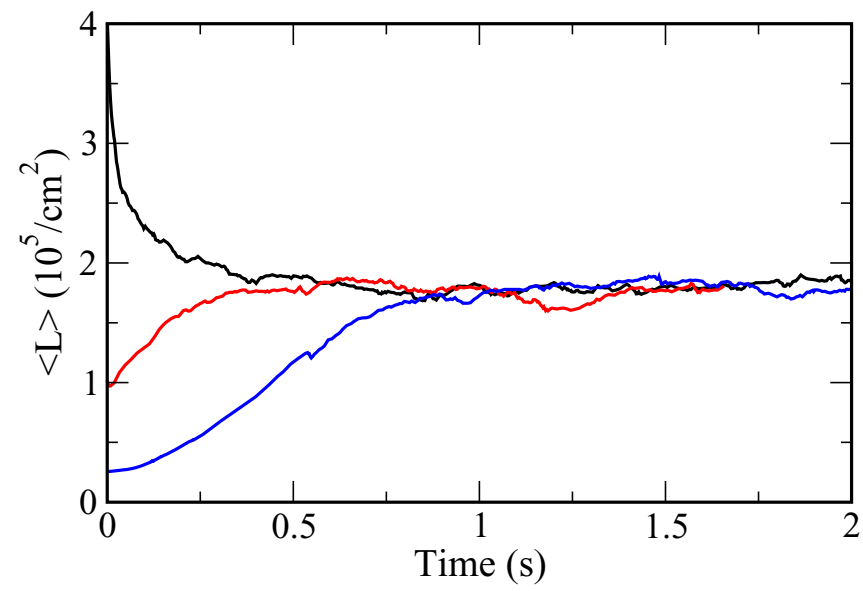

FIG. 12. Line length density as a function of time, for $v_{n s}=$ $1.4 \mathrm{~cm} / \mathrm{s}$ and nonlocal interactions over the entire volume, for three different initial configurations.

increasing vortex line length produced both an increase in polarization and a decrease in the reconnection rate exponent. Tsubota et al. also found a hint of a reduced exponent [42], albeit without enough data to identify the exponent. In Fig. 11 we fit reconnection rate vs velocity, using only the points corresponding to the four largest driving velocities, where the anisotropy parameter is changing little. We find that the exponent is indeed reduced, to 1.98. The resulting fit does not extrapolate well to the data from lower driving velocity, but that is not surprising since the low-velocity tangles are much more anisotropic, as seen in Fig. 9.

Both the average vortex line density and the tangle anisotropy are independent of the initial configuration used. For example, Fig. 12 shows that line density for $v_{n s}=$ $1.4 \mathrm{~cm} / \mathrm{s}$ ultimately reaches the same stable level for three initial conditions. The convergence to a common global line density happens despite the inhomogeneity among orthants. This demonstrates that equilibration occurs even with weak direct communication among different parts of the space.

\section{LOCAL INDUCTION APPROXIMATION}

A calculational issue on the 3-torus has been the reliability of the local induction approximation (LIA) that ignores the nonlocal integral in the equation of motion. Without the nonlocal term, the tangle can degenerate into an "open-orbit" state, consisting of straight parallel vortex lines that do not interact $[7,8]$. Keeping the nonlocal term solves this problem [9], but given the extra computational demands of the full integral even subsequent work has used LIA [43,44], compared results from LIA and the full integral $[13,45]$, or drawn conclusions from prior LIA work [46]. Hence better understanding its limitations remains relevant.

The flow lines of the Hopf field on the 3-sphere do not remain mutually perpendicular throughout the entire space, so we observe nothing directly equivalent to the open-orbit state on the 3-torus. However, omitting the nonlocal interaction causes a different problem: a collapse of vortex lines until they all lie nearly atop each other. In most cases the vortices in the collapsed state follow roughly along a Hopf fiber, 
(a)

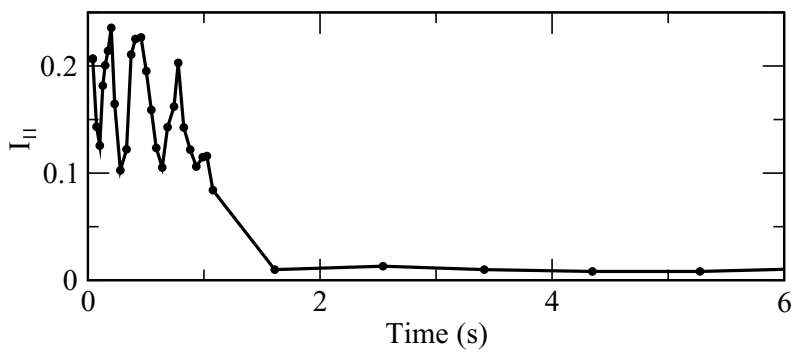

(b)

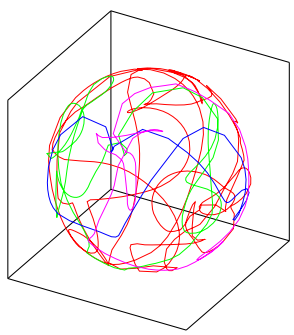

(d)

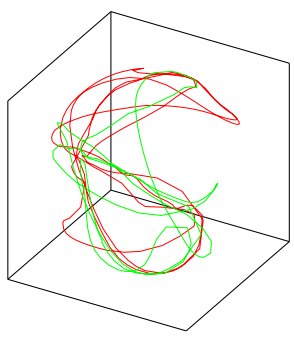

(f)

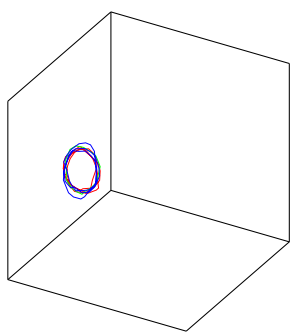

(c)

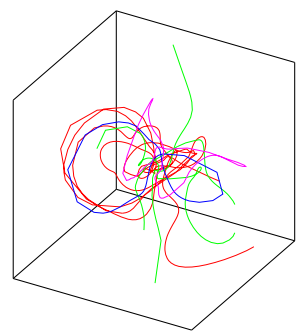

(e)

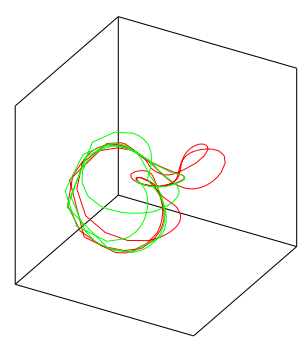

(g)

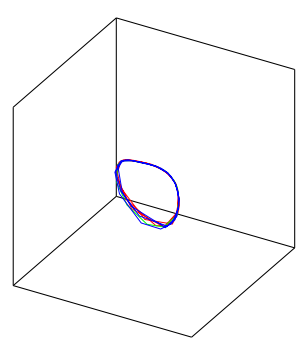

FIG. 13. Development of vortices for $v_{n s}=1.4 \mathrm{~cm} / \mathrm{s}$, with no nonlocal contribution. Frame (a) shows the anisotropy density as a function of time. Frames (b), (d), and (f) are Hopf projections of the vortices at $t=0,0.5$, and $1.6 \mathrm{~s}$, all with the same scale. Frames (c), (e), and (g) are stereographic projections at the same times, again all with the same scale.

leading to an extremely low anisotropy parameter. Figure 13 illustrates the collapse process at a driving velocity $v_{n s}=$ $1.4 \mathrm{~cm} / \mathrm{s}$. The first frame shows the anisotropy parameter which initially varies between 0.1 and 0.2 and then drops abruptly to about 0.01 , with little change thereafter. The remaining frames are Hopf and stereographic projections at three times. The initial configuration is a tangle equilibrated at the same driving velocity but with nonlocal terms included. As seen from frames (d) and (e), upon omitting the nonlocal contributions the vortex line density becomes less uniform, even before any dramatic change in the anisotropy appears. The final frames (f) and (g) show the complete collapse. Since the Hopf projection sends Hopf fibers to points, the
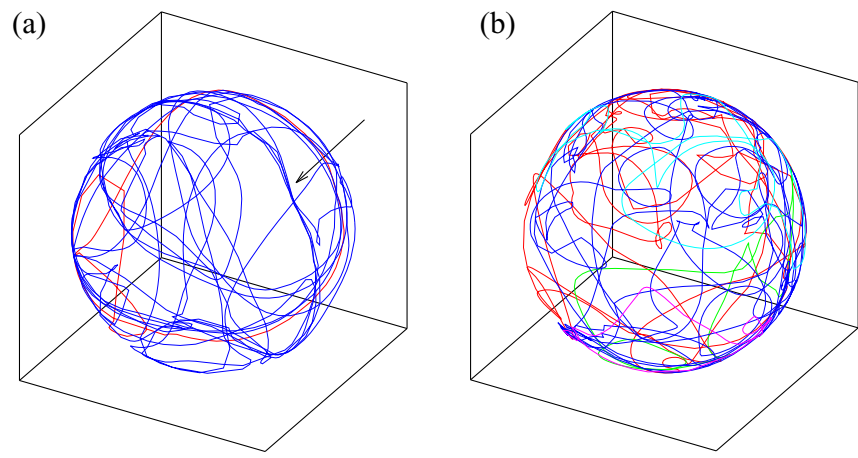

FIG. 14. Hopf projections for tangles generated with no nonlocal contributions (a) and with nonlocal contributions included out to a distance $d_{N L}=0.1 \pi R(\mathrm{~b})$. For both tangles $v_{n s}=2 \mathrm{~cm} / \mathrm{s}$ and the 3 -sphere radius is $R=0.005 \mathrm{~cm}$. Vortex clustering appears in (a), as indicated by the arrow, but is mostly eliminated in (b) by the shortdistance nonlocal contributions.

small size of the ring in (f) indicates that the vortices stray little from a single Hopf fiber. In frames (f) and (g) there are three distinct vortices present, which make a combined total of seven circuits. Continuing the calculation leads to trivial reconnections among these near-parallel vortices, but no other interactions occur and the collapsed state persists indefinitely.

Low-velocity calculations without the nonlocal contribution routinely collapse to a set of nearly overlapping loops, composing one or more vortex rings. While the loops are usually close to a Hopf fiber, with anisotropy parameter $I_{\|}<0.02$, that is not always the case. One of our collapsed configurations had final anisotropy parameter an order of magnitude larger, $I_{\|}=0.27$. For our usual right-handed Hopf flow, the vortex loop is roughly aligned with the flow. Starting with what appears to be a stable configuration and inverting the direction of all vortices causes immediate vortex motion, as the loops rotate by $\pi$ relative to the Hopf flow. Similarly, for a left-handed Hopf flow stable vortices are antiparallel to the field. This direction change is exactly as expected since vortices are axial vectors. Oddly, the persistence of such vortex arrangements depends on having several loops; upon removing all but a single loop, that loop moves and distorts until eventually additional loops are created. The requirement of multiple loops is particularly strange given that the LIA calculation does not include nonlocal interactions, so the loops interact solely through reconnections. The collapsed state appears to be a numerical artifact specific to using the local approximation on $S^{3}$, much as the open-orbit state is on $T^{3}$.

For sufficiently large driving velocity, a nontrivial vortex tangle persists even without nonlocal contributions, as illustrated in Fig. 14(a). However, hints of the collapsed state remain, in the tendency of vortices to clump together. Without the nonlocal term to separate them, portions of vortices that become aligned will continue their time development together until disrupted by reconnection with a nonaligned vortex. As with the open-orbit state on $T^{3}$, adding nonlocal contributions out to a very limited distance disrupts the collapsed state. The tangle of Fig. 14(b), which results from including contributions out to a distance $d_{N L}=0.1 \pi R$, shows little sign of 


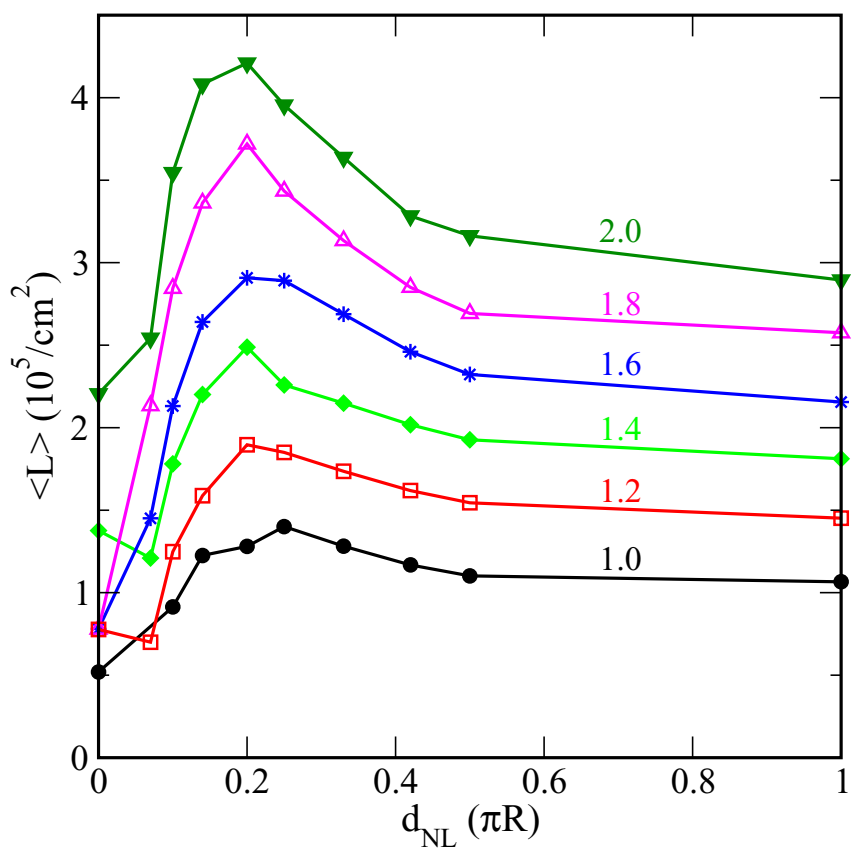

FIG. 15. Equilibrated line length density as a function of the extent of the nonlocal calculation, for several applied velocity fields. The velocities, in $\mathrm{cm} / \mathrm{s}$, are indicated above each curve.

the clustering that results from the purely local calculation. Our calculations suggest that, with nonlocal terms included even for such a small fraction of the 3-sphere, the tangles can persist indefinitely.

\section{EFFECT OF NONLOCAL TERM}

As with our previous simulations with the usual periodic boundary conditions [11], we examine the effects of including nonlocal terms only for vortices within a distance $d_{N L}$. Figure 15 shows the results. At very small $d_{N L}$, the main effect is to dissipate the clumping, since intervortex interactions separate the vortex lines. Forcing the vortices apart also helps to maintain a true tangle. Without the nonlocal interaction, the vortices can collapse along a flow line, which prevents further energy gain or loss in the tangle. The nonlocal term and resulting reconnections ensures that some of the vortex loops have segments perpendicular to the velocity field. These perpendicular segments exchange energy with the applied field, allowing the line density to grow. As $d_{N L}$ increases further, the line length density of the tangle decreases gradually. This is analogous to our previous results for periodic boundary conditions, where the nonlocal interaction reduces the line length by favoring reconnections between antiparallel vortices over those between near-parallel vortices. However, with periodic boundary conditions this decrease in $\langle L\rangle$ occurs mainly when $d_{N L}$ is smaller than the typical vortex separation. At larger $d_{N L}$ the line length is nearly constant. Effectively, only "nearest-neighbor" vortices contribute significantly to the velocity field. The much more gradual effect of nonlocal terms in $S^{3}$ may arise from the anisotropic nature of the tangles, which means that distant nonlocal contributions are not directed randomly and do not cancel as efficiently as they do on the 3-torus. Interestingly, the crossover between the

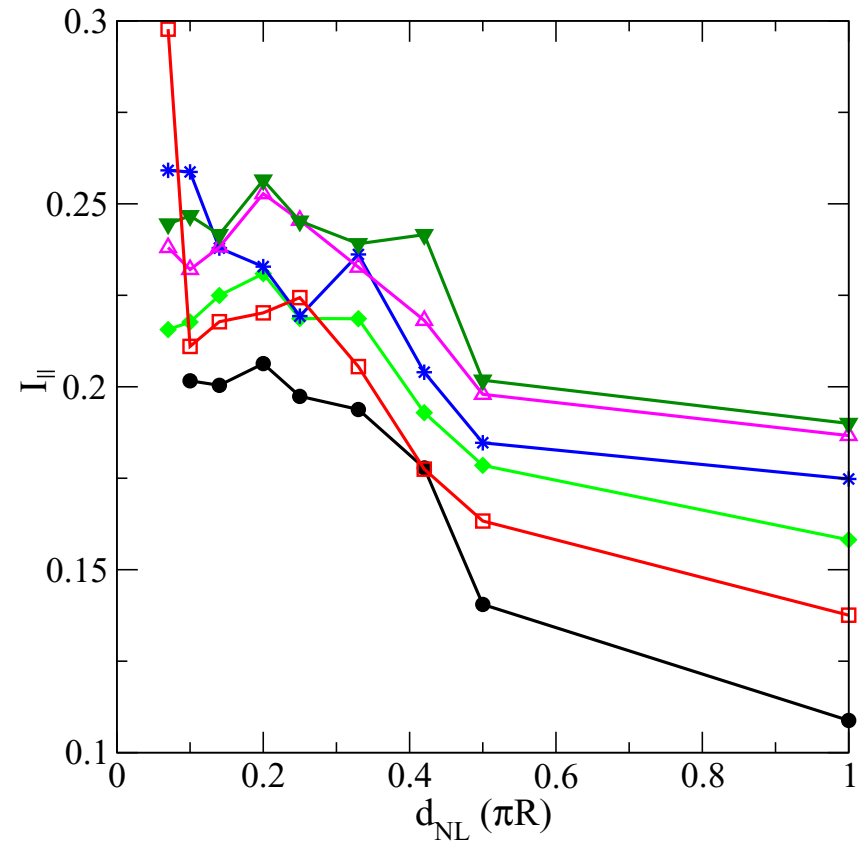

FIG. 16. Anisotropy parameter as a function of the extent of the nonlocal calculation, for several applied velocities. The driving velocities, in $\mathrm{cm} / \mathrm{s}$, are $1.0,1.2,1.4,1.6,1.8$, and 2.0 , with $I_{\|}$strictly increasing with drive at $d_{N L}=1$. (This is the same color scheme as in Fig. 15.)

low- $d_{N L}$ reduction of clumping and the high- $d_{N L}$ reduction of line length occurs when the nonlocal term is applied over a distance comparable to the vortex line spacing. In fact, the maximum line length density seems to move towards smaller $d_{N L}$ as the applied velocity field increases, consistent with the reduced line spacing at higher velocities.

As shown in Fig. 16, the tangles become slightly more anisotropic as the driving velocity decreases, probably because at lower line density there are fewer reconnections. The anisotropy is relatively constant at low $d_{N L}$, but increases as the interactions extend beyond one hemisphere. This increased anisotropy is entirely different from the collapse observed as $d_{N L} \rightarrow 0$, and much more subtle. The vortices remain intertwined, but with an incomplete tendency to align with the velocity field. The increase in this alignment when the interactions reach a full hemisphere suggests that the coupling of distant regions through the full $S^{3}$ Biot-Savart law plays a significant role in the anisotropy. In fact, extending the interactions from one hemisphere to the entire 3 -sphere affects the anisotropy more than it does the line density. Figure 16 omits combinations of $d_{N L}$ and $v_{n s}$ for which the tangle collapses to a single trajectory of nearly overlapping vortices. At the lowest $d_{N L}$, where the vortices do not completely collapse, $I_{\|}$ relies heavily on a small number of vortex loops and becomes erratic, for example, at $v_{n s}=1.2 \mathrm{~cm} / \mathrm{s}$ and $d_{N L}=0.07 \pi R$.

\section{POLARIZATION OF TANGLES}

We now return to the significant difference in tangle anisotropy between simulations on $T^{3}$ and $S^{3}$. In 3-torus calculations with periodic boundary conditions we find a 

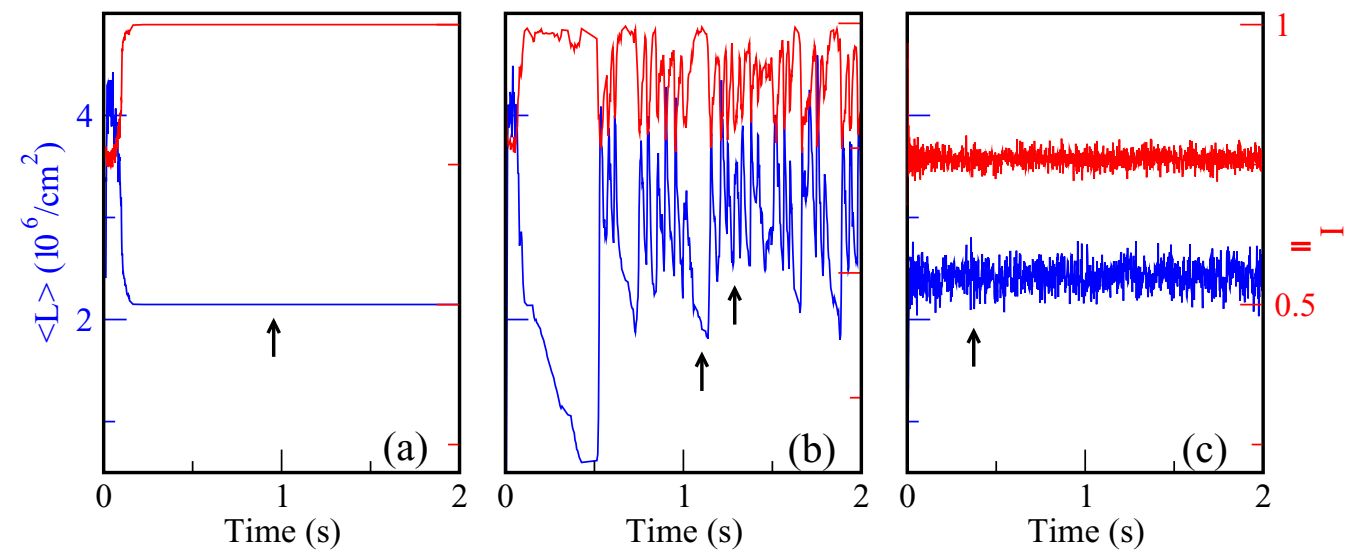

FIG. 17. Line length density (lower curves, left axis) and anisotropy (upper curves, right axis) on a 3 -torus, with $v_{n s}=12 \mathrm{~cm} / \mathrm{s}$. (a) LIA only, straight velocity field; (b) LIA only, helical velocity field; (c) full Biot-Savart law, straight velocity field. Arrows indicate the configurations shown in Fig. 18.

slight tendency of vortices to align perpendicular to the driving velocity field. The Glaberson-Donnelly instability [47], which has been observed by various groups both experimentally $[28,48]$ and in computational work $[37,49]$, prevents parallel alignment. Although a straight vortex parallel to the velocity is a solution to the equations of motion, it is unstable. If a fluctuation in the vortex core position produces a bulge with positive velocity flux through it, then that bulge will grow. In particular, for a vortex aligned with the velocity, i.e., with circulation following the righthand rule about the velocity field, a right-handed helical distortion grows but a left-handed helix collapses back to a straight line. With multiple vortices, the growth of unstable distortions ultimately leads to reconnections that alter the vortex arrangement. The Glaberson-Donnelly instability also impacts simulations using the local induction approximation, where an "open-orbit" state appears which has noninteracting vortices aligned in a single direction. The instability ensures that the alignment direction is perpendicular to the driving velocity.

By contrast, on $S^{3}$ the driving velocity field follows helical Hopf fibers. No set of parallel vortices, all perpendicular to the velocity field throughout the 3-sphere, can exist, so a vortex tangle never collapses to an open-orbit state as in $T^{3}$. Furthermore, the Glaberson-Donnelly instability itself is weakened. One clue as to why is that the vortex rings in our simulations can only align along the Hopf flow with a particular handedness. Our usual Hopf flow is right-handed, with its curl in the same direction as the original flow, and vortices align with the flow. For a left-handed Hopf flow, with curl opposing the flow, stable vortices must oppose the field. As in $T^{3}$, a vortex aligned with the velocity field is stable to left-handed helical distortions. If the Hopf field is left-handed, then a right-handed helical distortion grows, again as in $T^{3}$. However, for a right-handed Hopf field, the longest-wavelength right-handed helical distortion is itself another flow line. Such a perturbation has no tendency to grow or to shrink; the distortion leaves the vortex aligned with the velocity field. For LIA calculations in $S^{3}$ we find near-perfect alignment with the Hopf flow. The polarization decreases once nonlocal interactions are included, although the turbulence remains anisotropic with a tendency towards parallel alignment.

This raises the question of whether a helical velocity field on a 3-torus would lead to some of the same behavior as the Hopf flow on the 3-sphere. We carried out a few such simulations, using a right-handed helical field around the $z$ axis. The field makes three rotations and has horizontal magnitude $10 \%$ of the total. The helical field indeed prevents the full open-orbit state from developing even when using the local induction approximation. However, the vortex line density still differs from that of the full nonlocal calculation. Figure 17 shows the relatively stable line density from a full nonlocal calculation, along with the perfectly stable line density from LIA once the open orbit state has formed. The exact open-orbit line density varies depending on the initial conditions; in this case it is slightly lower than that of the nonlocal calculation. The helical velocity field leads to entirely different behavior. The tangle undergoes frequent excursions towards the open-orbit state, with the anisotropy coefficient becoming very large; Fig. 18(c) illustrates a configuration with $I_{\|}=0.992$ and $\langle L\rangle=1.9 \times 10^{6} \mathrm{~cm}^{2}$. Unlike in the complete open-orbit state of Fig. 18(a), slight curvature and misalignment remain. Remarkably, the helical velocity field enables recovery even from such a well-aligned configuration. A short time later, the vortices reach the configuration of Fig. 18(d), with $I_{\|}=0.787$ and $\langle L\rangle=3.4 \times 10^{6} \mathrm{~cm}^{2}$. The tangle remains noticeably less homogeneous than that of Fig. 18(b), which comes from a fully nonlocal calculation and has $I_{\|}=0.787$ and $\langle L\rangle=2.6 \times 10^{6} \mathrm{~cm}^{2}$. Figure $17(\mathrm{~b})$ shows repeated fluctuations between these limits. The dependence on the helicity of the applied velocity field is entirely an effect of LIA; a full nonlocal calculation with helical applied velocity has density and anisotropy unchanged from Fig. 17(c).

\section{CONCLUSIONS}

We have carried out vortex simulations on a 3-sphere. Our results show the potential importance of the global geometry and topology used. The most basic features remain the same as in standard 3-torus-based calculations. Vortex tangles appear, with the vortex line length stabilizing at a value that increases 
(a)

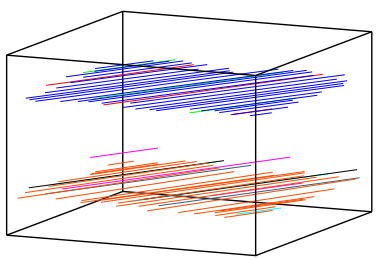

(c)

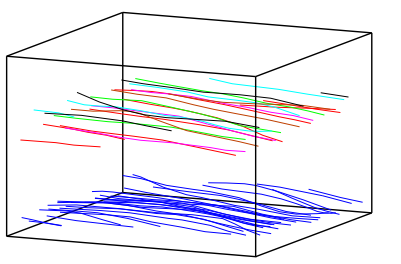

(b)
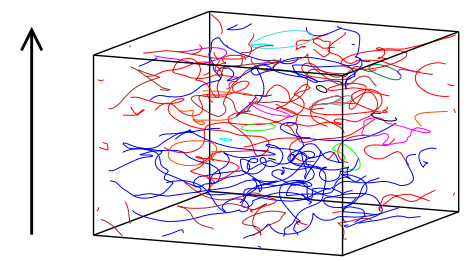

(d)

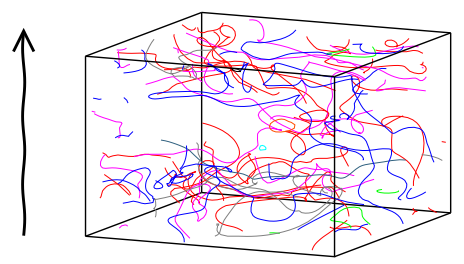

FIG. 18. Vortex configurations for 3-torus simulations, corresponding to the points noted in Fig. 17. (a) LIA only, straight velocity field; (b) full Biot-Savart law; (c) LIA only, helical velocity field, high-anisotropy configuration; (d) LIA only, helical velocity field, low-anisotropy configuration. The black lines between the graphs track a flow line of the velocity field for the straight and helical cases. As indicated, the horizontal velocity within the helix is only $10 \%$ of the vertical velocity.

with the driving velocity. However, the tangles are much less isotropic than those generated on a torus. Exponents relating the line length to the driving velocity and the reconnection rate reflect this increased anisotropy. This suggests the intriguing possibility of generating steady-state tangles with markedly different properties by changing the underlying global structure.

In calculations using the local induction approximation, the influence of the manifold was even more striking. The particular failure mode on $T^{3}$, the open orbit state, did not appear, but vortices instead collapsed to occupy a thin tube, all with nearly parallel alignment. A helical driving velocity on $T^{3}$, which shares some properties with the flow on $S^{3}$, avoids the full open orbit state under LIA, but the tangles it generates retain other characteristics of those generated on $T^{3}$ by a straight driving velocity. This is further evidence of the role of the manifold in such calculations.

A key question for any physics simulation is how well it matches physical reality. A 3-torus is standard for computations throughout condensed matter, as a convenient way to explore a finite volume without boundaries. Yet our results show that global features of the manifold used in calculations can significantly impact the results. Disturbingly, except for properties which depend on the curvature of space, there is no a priori reason to assume that a 3 -torus will produce the best approximation to behavior in $\mathbb{R}^{3}$. We recommend that more attention be paid to possible effects from the topology of the space selected for a computation.

\section{ACKNOWLEDGMENTS}

We thank G. Kuperberg for many helpful conversations, including bringing to our attention the exact Biot-Savart formula on $S^{3}$. One of us (O.M.D.) acknowledges funding from a Department of Education GAANN fellowship.

\section{APPENDIX \\ 1. Stereographic projection}

In this case $S^{3}$ is mapped onto $\mathbb{R}^{3}$. Figure 19 shows the analogous operation mapping $S^{2}$ to the $z=0$ plane. The projection point $p$ is $(0,0, R)$. A line is extended from $p$ through a point on the circle, and the intersection between that line and the $x y$ plane gives the image point. Under this projection, the equator maps to a circle of radius $R$. The lower hemisphere goes to the inside of this circle and the outer hemisphere to the outside. The projection point itself does not have an image within the plane, but maps to a point at infinity. In general, the image of a point $(x, y, z)$ in $S^{2}$ has coordinates

$$
\left(\frac{x}{1-z / R}, \frac{y}{1-z / R}\right)
$$

in the plane. The generalization to a projection from $S^{3}$ to, say, the $w=0$ space is straightforward.

The main disadvantage of stereographic projection is that distances are not preserved. One half of $S^{3}$ maps to the inside of a finite sphere, while the other half maps to the infinite outside. This can give a misleading impression of the structure of a vortex tangle.

\section{Hopf fibration}

A Hopf fibration maps $S^{3}$ onto $S^{2}$ in a way that preserves distance, up to an overall scale factor. It is selected to match our driving velocity field, $\mathbf{v}=\frac{v}{R}(-y, x,-w, z)$; as we show below, the corresponding Hopf fibration maps each flow line to a single point. We take the Hopf fibration as the following map to $\mathbb{R}^{3}$ :

$$
\begin{array}{r}
h_{1}=2(x z+y w), \\
h_{2}=2(y z-x w), \\
h_{3}=\left(x^{2}+y^{2}\right)-\left(z^{2}+w^{2}\right) .
\end{array}
$$

Explicit multiplication shows that, for any point on a 3 -sphere of radius $R$, its image is at radius $R^{2}$. Thus $S^{3}$ in fact maps to $S^{2}$.

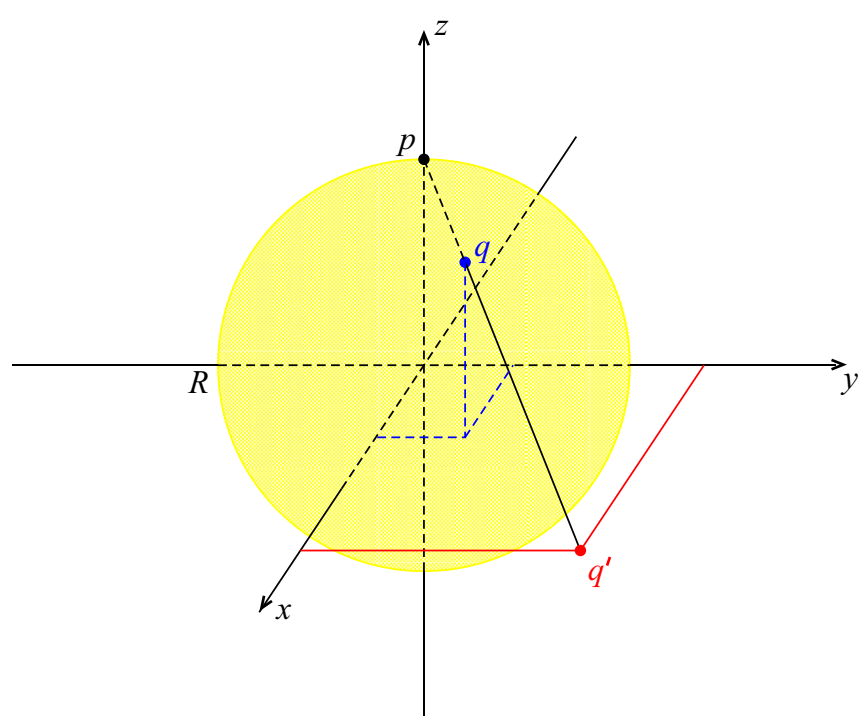

FIG. 19. Illustration of stereographic projection from the surface of a three-dimensional ball to the $x y$ plane. A point $q$ maps to $q^{\prime}$ in the plane such that $q, q^{\prime}$, and $p$ are collinear. 
The flow lines of the velocity field are particular great circles on $S^{3}$. To track them under the Hopf fibration, consider the four-dimensional real space which contains $S^{3}$ as a two-dimensional complex space with coordinates $q_{1}=x+i y$ and $q_{2}=z+i w$. A complex line, $q_{2}=k q_{1}$, intersects $S^{3}$ in a great circle of constant $\left|q_{1}\right|$ and $\left|q_{2}\right|$, which is exactly a flow line of this velocity field. In addition, writing the Hopf fibration in terms of the complex coordinates and using $q_{2}=$ $k q_{1}$ gives

$$
\begin{array}{r}
h_{1}=\operatorname{Re}\left(2 q_{1} q_{2}^{*}\right)=2\left|q_{1}\right|^{2} \operatorname{Re}(k), \\
h_{2}=\operatorname{Im}\left(2 q_{1} q_{2}^{*}\right)=2\left|q_{1}\right|^{2} \operatorname{Im}(k), \\
h_{3}=\left|q_{1}\right|^{2}-\left|q_{2}\right|^{2}=\left|q_{1}\right|^{2}\left(1-|k|^{2}\right) .
\end{array}
$$

The image depends only on $k$ and $\left|q_{1}\right|$, both of which are constant on any great circle corresponding to a flow line of the velocity field. Hence this Hopf fibration maps a flow line on $S^{3}$ to a point on $S^{2}$.

Any two Hopf fibers of the flow are great circles that twist around each other. The distance between any point on one fiber and the closest point on the other is constant, which gives a sensible definition for the distance between the two fibers. The Hopf fibration preserves this distance, up to an overall scale factor. For example, all fibers a fixed distance from a given fiber $A$ are mapped to a circle on $S^{2}$ centered at the image of $A$.
[1] J. Blazek, Computational Fluid Dynamics, Principles and Applications, 3rd ed. (Elsevier Ltd., Amsterdam, 2015).

[2] M. Maxey, Annu. Rev. Fluid Mech. 49, 171 (2017).

[3] M. L. Hosain and R. B. Fdhila, Energy Proc. 75, 3307 (2015).

[4] G.-H. Cottet and P. D. Koumoutsakos, Vortex Methods: Theory and Practice (Cambridge University Press, Cambridge, UK, 2000).

[5] K. W. Schwarz, Phys. Rev. B 31, 5782 (1985).

[6] M. Tsubota, K. Fujimoto, and S. Yui, J. Low Temp. Phys. 188, 119 (2017)

[7] K. W. Schwarz, Phys. Rev. B 38, 2398 (1988).

[8] R. G. M. Aarts, Ph.D. thesis, Eindhoven University of Technology, 1993.

[9] H. Adachi, S. Fujiyama, and M. Tsubota, Phys. Rev. B 81, 104511 (2010).

[10] C. Barenghi and D. Samuels, J. Low Temp. Phys. 136, 281 (2004).

[11] O. M. Dix and R. J. Zieve, Phys. Rev. B 90, 144511 (2014).

[12] M. Tsubota, T. Araki, and S. K. Nemirovskii, Phys. Rev. B 62, 11751 (2000).

[13] L. Kondaurova, V. L’vov, A. Pomyalov, and I. Procaccia, Phys. Rev. B 89, 014502 (2014).

[14] J. Tough, in Progress in Low Temperature Physics, edited by D. F. Brewer (North-Holland, Amsterdam, 1982), Vol. 8, p. 133.

[15] D. J. Melotte and C. F. Barenghi, Phys. Rev. Lett. 80, 4181 (1998).

[16] A. W. Baggaley and S. Laizet, Phys. Fluids 25, 115101 (2013).

[17] V. I. Arnold and B. A. Khesin, Topological Methods in Hydrodynamics (Springer-Verlag, New York, 1998), p. 127.

[18] M. P. do Carmo, Differential Geometry of Curves and Surfaces (Prentice-Hall, Englewood Cliffs, NJ, 1976).

[19] D. DeTurck and H. Gluck, J. Math. Phys. 49, 023504 (2005).

[20] G. Kuperberg, Geom. Funct. Anal. 18, 870 (2008).

[21] J. Koplik and H. Levine, Phys. Rev. Lett. 71, 1375 (1993).

[22] M. Tsubota and S. Maekawa, J. Phys. Soc. Jpn. 61, 2007 (1992).

[23] A. W. Baggaley and C. F. Barenghi, J. Low Temp. Phys. 166, 3 (2012).

[24] Y. Oshima and N. Izutsu, Phys. Fluids 31, 2401 (1988).

[25] D. Kleckner and W. T. M. Irvine, Nat. Phys. 9, 253 (2013).
[26] G. P. Bewley, M. S. Paoletti, K. R. Sreenivasan, and D. P. Lathrop, Proc. Natl. Acad. Sci. (USA) 105, 13707 (2008).

[27] W. P. Thurston, Three-Dimensional Geometry and Topology (Princeton University Press, Princeton, NJ, 1997).

[28] C. E. Swanson, C. F. Barenghi, and R. J. Donnelly, Phys. Rev. Lett. 50, 190 (1983).

[29] C. F. Barenghi, S. Hulton, and D. C. Samuels, Phys. Rev. Lett. 89, 275301 (2002).

[30] R. W. Stewart, Radio Sci. 4, 1269 (1969).

[31] A. La Port, G. A. Voth, A. M. Crawford, J. Alexander, and E. Bodenschatz, Nature (London) 409, 1017 (2001).

[32] G. E. Elsinga and I. Marusic, Phys. Fluids 28, 011701 (2016).

[33] L. Biferale, D. Khomenko, V. L'vov, A. Pomyalov, I. Procaccia, and G. Sahoo, Phys. Rev. Lett. 122, 144501 (2019).

[34] L. Biferale, D. Khomenko, V. L’vov, A. Pomyalov, I. Procaccia, and G. Sahoo, Phys. Rev. B 100, 134515 (2019).

[35] C. E. Swanson and R. J. Donnelly, J. Low Temp. Phys. 61, 363 (1985).

[36] K. P. Martin and J. T. Tough, Phys. Rev. B 27, 2788 (1983).

[37] M. Tsubota, T. Araki, and C. F. Barenghi, Phys. Rev. Lett. 90, 205301 (2003).

[38] D. R. Poole, H. Scoffield, C. F. Barenghi, and D. C. Samuels, J. Low Temp. Phys. 132, 97 (2003).

[39] S. K. Nemirovskii, Phys. Rev. Lett. 96, 015301 (2006).

[40] V. S. L'vov, S. V. Nazarenko, and O. Rudenko, Phys. Rev. B 76, 024520 (2007).

[41] J. Laurie and A. W. Baggaley, J. Low Temp. Phys. 180, 82 (2015).

[42] M. Tsubota, C. F. Barenghi, T. Araki, and A. Mitani, Phys. Rev. B 69, 134515 (2004).

[43] H. Salman, Phys. Rev. Lett. 111, 165301 (2013).

[44] R. A. Van Gorder, Phys. Fluids 25, 085101 (2013).

[45] J. Laurie and A. W. Baggaley, J. Low Temp. Phys. 180, 95 (2015).

[46] S. K. Nemirovskii, arXiv:1902.06214.

[47] W. I. Glaberson, W. W. Johnson, and R. M. Ostermeier, Phys. Rev. Lett. 33, 1197 (1974).

[48] D. K. Cheng, M. W. Cromar, and R. J. Donnelly, Phys. Rev. Lett. 31, 433 (1973).

[49] M. Mesgarnezhad, R. G. Cooper, A. W. Baggaley, and C. F. Barenghi, Fluid Dyn. Res. 50, 011403 (2018). 